\title{
Endotelio y mujer: similaridad y diferencias con el hombre
} Endothelium and woman: similitude and differences with man

\author{
Enrique Melgarejo R. ${ }^{1,2,3,4, a}$ \\ Profesor de Medicina, Universidad Militar Nueva Granada, Bogotá, Colombia. \\ ${ }^{2}$ Profesor Emérito, Hospital Militar Central. \\ ${ }^{3}$ Presidente Emérito, Colegio Panamericano del Endotelio. \\ ${ }^{4}$ Coordinador del Comité de Géneros, Razas y Minorías. \\ ${ }^{a}$ Doctor en Medicina, Cardiólogo-Electrofisiólogo.
}

\begin{abstract}
Resumen
Existen diferencias intrasexo en lo referente a función y disfunción endotelial. Aunque ambos sexos comparten los mismos receptores hormonales (estrogénicos y de testosterona), varian en su expresión. Los receptores estrogénicos en la mujer ejercen acción protectora vascular por vía genómica y no genómica, mediadas a través del óxido nítrico. La mujer está 'protegida' para la aterotrombosis hasta la menopausia. La pérdida abrupta de la protección vascular conlleva a magnificación del daño vascular posmenopausia, más si ya tiene disfunción endotelial. La mujer hasta la menopausia tiene un ambiente vascular menos oxidativo con relación al hombre. Las mujeres tienen más angina microvascular y menos infarto con elevación del segmento ST, debido a que hacen más disfunción endotelial ante los factores de riesgo. Las mujeres posmenopáusicas hacen más hipertensión sistólica y por consiguiente tienen mayor presión de pulso y rigidez arterial, esto último mediado por el óxido nítrico.
\end{abstract}

Palabras clave: Endotelio, endotelio y mujer, estrógenos y endotelio.

\section{Abstract}

There are intra-sexual differences in both endothelial function and dysfunction. Although both sexes share the same hormonal receptors (estrogen and testosterone receptors) these differ in expression. Female estrogen receptors exert vascular protective action through nitric oxide-mediated genomic and non-genomic pathways. Women are 'protected' from atherothrombosis until menopause. The abrupt loss of vascular protection leads to a magnification of post-menopausal vascular damage, which is higher when endothelial dysfunction is already present. Women have a less oxidative vascular environment until menopause than men. Women experience more microvascular angina and less myocardial infarction with ST segment elevation, because they display more endothelial dysfunction when risk factors are present. Post-menopausal women develop more prominently systolic hypertension and therefore higher pulse pressure and arterial stiffness, the latter mediated by nitric oxide.

Keywords: Endothelium, vascular sex differences, estrogens and endothelium.

An Fac med. 2014;75(4):339-43 / doi: http://dx.doi.org/10.15381/anales.v75i4.10855

\section{INTRODUCCIÓN}

Durante los últimos 18 años se ha gestado a nivel mundial una conciencia acerca de diferencias en la medicina de géneros y razas ${ }^{(1,2)}$. En algunos países o regiones son más marcadas estas diferencias, especialmente en lo referente a morbimortalidad cardiovascular, y en otros, el problema ha variado a través de los últimos años (figura 1).

Es la cardiología el área de la salud en la cual se ha encontrado mayores diferencias en lo referente a los géneros ${ }^{(4,5)}$. Esto simplemente porque, como especialidad y funcionalidad, involucra a todos los tejidos irrigados, incluyendo obviamente los sistemas neuro-renocardio-vascular, a través del templo de la pared vascular. Es precisamente en esta estructura en donde hay diferencias genómicas entre géneros que inciden directamente y con impacto clínico, mediado a través de diferencias en receptores, carga enzimática, canales, ligandos, caveolas, matriz extracelular, entre otros.

Estas diferencias se inician desde la vida fetal, en la etapa de diferenciación, con cambios fenotípicos. Posteriormente, en la vida extrauterina se presenta una segunda etapa a partir de la adolescencia, en la cual se acoplan las fluctuaciones y variaciones cíclicas hormonales en la mujer, con sus efectos asociados durante los ciclos menstruales y de ovulación, los embarazos, durante el paso casi abrupto de la de la vida fértil a la menopausia y durante la posmenopausia ${ }^{(6)}$.

\section{ORÍGENES}

Desde el punto de vista genómico, la carga genética de los homínidos fue hecha para ser una especie nómada, competitiva y hasta guerrera. Pero, a través de los millones de años de evolución y 


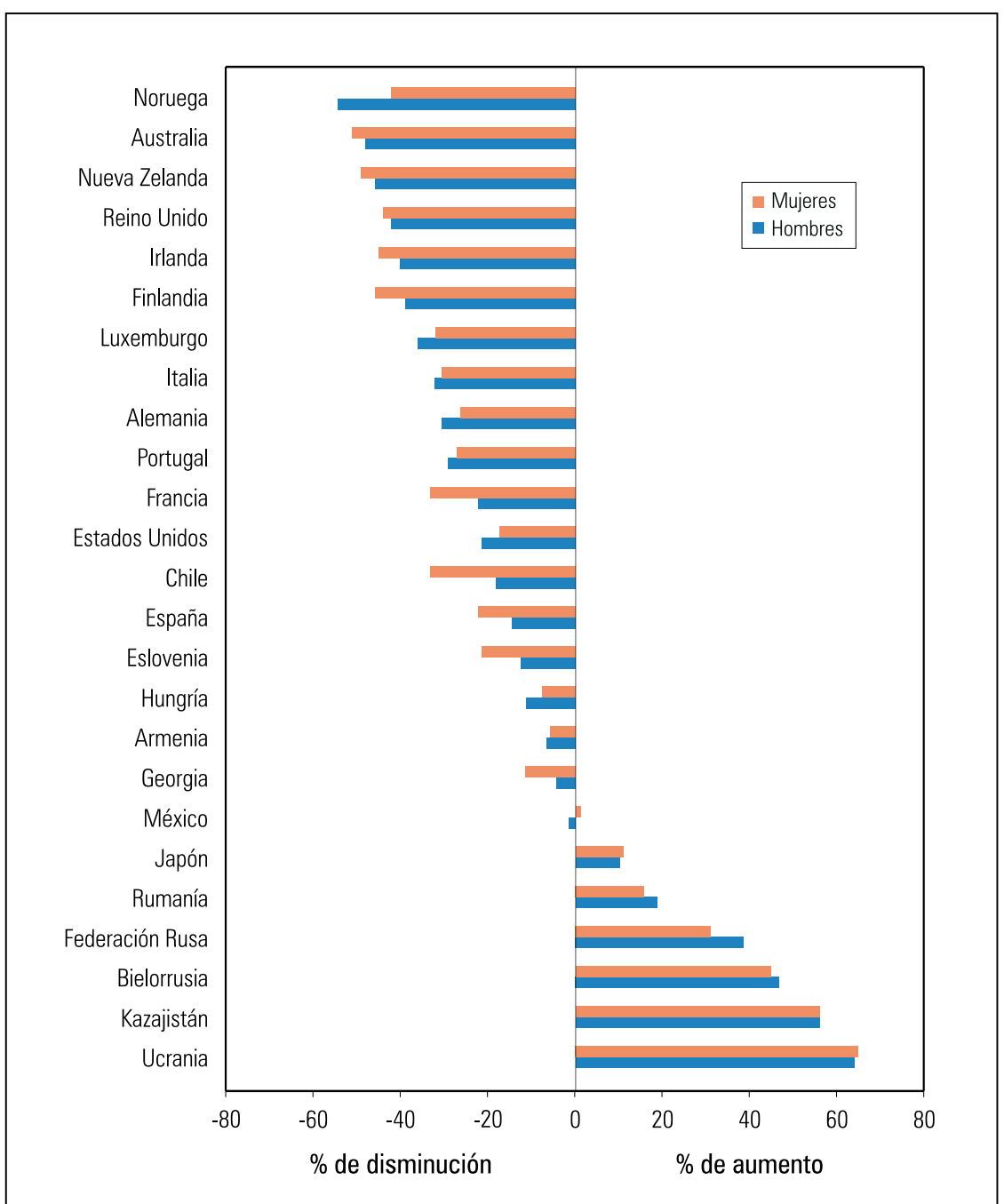

Figura 1. Modificaciones en las tasas de mortalidad cardiovascular entre hombres y mujeres de 35 a 74 años de edad, entre 1990 y 2000 (OMS) ${ }^{(3)}$.

los diferentes procesos antropológicos y culturales por los cuales ha cursado la humanidad, se pasó de una era nómada a una era urbana, industrializada y eminentemente sedentaria, en donde las máquinas tienden cada vez más a hacer el trabajo de los hombres. El genotipo no ha variado. Sigue siendo el mismo. Pero hemos cambiado el fenotipo competitivo hacia el fenotipo 'vulnerable'. Vulnerable precisamente a procesos patológicos secundarios a estos cambios de comportamiento o de estilo de vida. Y ¿por qué inciden en este nivel? Porque es en la vasculatura -pared arterial- en donde se inicia el depósito de las grasas saturadas que el humano empezó a ingerir y para lo cual ontogénicamente no veníamos programados ni preparados como especie. Al cambiar sus costumbres, el ser humano, al dejar de ser pescador, pastor y cazador y al empezar a ingerir grasas saturadas y tornarse sedentario, se empezaron a acumular las grasas saturadas y trans (estas últimas con un potente efecto pro-oxidante en la pared arterial), iniciándose el proceso de la aterosclerosis y la tendencia y riesgo hacia la aterotrombosis.

\section{¿Dónde radican las diferencias?}

La vasculatura de la mujer a nivel endotelial expresa receptores estrogénicos, los cuales facilitan la producción de óxido nítrico $(\mathrm{NO})$-mucho más que el hombre-, pero se pierde esta acción y esta 'protección natural' si es fumadora, diabética o usa inadecuadamente hormonas sexuales, incluyendo algunos anovulatorios.

Una entidad que se ve más frecuentemente en mujeres que en hombres, como es el shock séptico, en este hay una hiperproducción de óxido nítrico (NO), por la vía inducible y no por la constitutiva, generando vasodilatación severa e irreversible y una verdadera plejia arterial. En la eclampsia también hay un alto nivel oxidativo. Es decir, estas entidades corresponden a patologías endoteliales específicas de la mujer ${ }^{(7)}$.

\section{Diferencias sexuales en la función y disfunción endotelial}

Existen diferencias en cuanto al tipo de fisiopatología, manifestaciones y pronóstico, diferentes de las enfermedades cerebro-cardio-reno-vasculares en la mujer. Y estas diferencias están marcadas por la función y por la disfunción de las células endoteliales ${ }^{(8)}$. Las mujeres hacen mayor disfunción microvascular que los hombres ${ }^{(9,10)}$. Es así como la enfermedad coronaria en la mujer es más 'microvascular', lo cual explica el por qué hacen más angina que infarto con relación a los hombres (estos hacen más infarto con elevación del segmento ST) ${ }^{(11)}$.

Las mujeres con infarto tienen más coronarias epicárdicas 'sanas' desde el punto de vista angiográfico. Esto se traduce como mayor disfunción endotelial o más enfermedad de pequeños vasos. Lo anterior también explica la diferencia de síntomas anginosos o isquémicos entre mujeres y hombres (más dolor epigástrico o dorsal en las mujeres).

Y el infarto se presenta en la menopausia, más prevalentemente después de los 65 años. Las mujeres se infartan 10 años después que el hombre -salvo que como ya se mencionó sea fumadora o diabética-. Está demostrado que durante la menopausia (tanto en normotensas como en hipertensas) hay alteración de vasodilatación mediada 
por flujo. De esta manera, el daño vascular se magnifica progresivamente en la mujer que ya tiene daño o disfunción endotelial cuando llega a la menopausia. Por el contrario, la mujer que llega a la menopausia con su vasculatura 'sana', está más protegida y desarrollará mucho menos probabilísticamente enfermedad aterotrombótica.

Esto trae consecuencias terapéuticas, pues la suplencia estrogénica al inicio de la menopausia, si no hay daño vascular, sí puede ser empleada y no genera daño cardiovascular siempre y cuando no se la utilice por más de 10 años. Otra historia es si hay riesgo o carga genética para cáncer de seno.

En el endotelio vascular de las mujeres y los hombres se encuentran receptores para estrógenos, progesterona y testosterona, al igual que en músculo liso, macrófagos y plaquetas. Pero varían en su expresión, mas no en su función ${ }^{(12)}$.

\section{PROTECCIÓN VASCULAR POR EL ENDOTELIO FEMENINO}

Hay evidencia que demuestra que el óxido nítrico $(\mathrm{NO})$ basal es mayor en mujeres que en hombres ${ }^{(13)}$. También hay evidencia que los lechos vasculares son diferentes. Por ejemplo, la vasculatura renal de los hombres es más dependiente del $\mathrm{NO}$ con la edad y comparado con las mujeres, indicando que cualquier enfermedad renal que interfiera con la producción del $\mathrm{NO}$ puede, a través del tiempo, generar mayor daño y progresar más rápidamente con relación al sexo opuesto ${ }^{(14)}$.

Igualmente el medio pro-oxidante es menos pronunciado en mujeres, ya que ellas tienen menor expresión de la NADPH oxidasa ${ }^{(15)}$ y de marcadores de estrés oxidativo ${ }^{(16,17)}$.

Además, los hombres in vivo tienen una mayor inactivación del NO. Se ha encontrado una mayor concentración del inactivador de la NO sintasa constitutiva o endotelial: la ADMA (asymmetrical dimethyl-L-arginine) ${ }^{(18)}$.
Otra diferencia importante está dada por la diferente respuesta a la endotelina (ET-1) al igual que sus concentraciones, siendo mayor en el hombre ${ }^{(19)}$.

Experimentalmente, la evidencia sugiere que el $\mathrm{NO}$ es más importante en las pequeñas arterias para producir vasodilatación en las hembras, mientras que el NO tiene un papel más preponderante en las arterias de los machos, demostrado en varios lechos vasculares (mesentérico, y cola, en ratas) ${ }^{(20-22)}$.

\section{EL PAPEL DE LOS ESTRÓGENOS}

Los estrógenos además de marcar diferencias fenotípicas, lo hacen también en la fisiopatología vascular. Como ya se mencionó, ejercen una acción antioxidante, lo cual permite prolongar la vida media del $\mathrm{NO}$ que es de tan solo 5 segundos. El anillo aromático presente en los estrógenos -y que está ausente en la progesterona y andrógenos- actúa como una variedad independiente de antioxidante (independiente del NO, que es un potente antioxidante) ${ }^{(23)}$.

Aún no hay claridad sobre la relación de los estrógenos y la prostaciclina $\left(\mathrm{PgI}_{2}\right)$ y su efecto vasodilatador y antiagregante plaquetario. Hay estudios contradictorios positivos ${ }^{(24-26)}$ y negativos ${ }^{(27,28)}$.

Los estrógenos logran su efector protector a través de modificaciones funcionales, reversible o irreversibles, de la composición de la membrana basal, canales iónicos (especialmente calcio y potasio), transducción de señales, logrados a través de receptores ${ }^{(29)}$.

Un hecho interesante es que la deficiencia de estrógenos en el hombre ha sido asociada con riesgo cardiovascular aumentado ${ }^{(30,31)}$. Se ha demostrado que la testosterona en los hombres y otras especies produce vasodilatación, pero por una vía endotelial independiente, involucrando la activación de los canales de calcio o inhibiendo los canales de potasio ${ }^{(32-34)}$.

Igualmente, se ha demostrado una alteración de la vasodilatación mediada por el endotelio en pacientes con hipogonadismo. Pero, la administración de testosterona no mejora la vasodilatación, y por el contrario empeora la disponibilidad del $\mathrm{NO}^{(35)}$.

Desde tiempo atrás se ha demostrado que la administración o suplencia con testosterona puede aumentar el riesgo cardiovascular. Pero, esto depende de las condiciones cardiovasculares preexistentes, de la edad (mayor en ancianos), la duración del tratamiento o el territorio vascular estudiado.

Por otro lado, los estrógenos pueden influenciar el sistema vascular del hombre. La administración aguda de estradiol a hombres jóvenes, en una concentración similar a la de las mujeres menopáusicas, induce vasodilatación vía endotelial ${ }^{(36)}$, incluyendo el lecho coronario. Que esto tenga implicaciones terapéuticas para un futuro, es tema aún controversial.

Por otro lado, la administración a largo plazo de estrógenos en transexuales hombre-mujer mejora la vasodilatación mediada por flujo ${ }^{(37)}$.

\section{El papel de los subtipos de receptores estrogénicos}

Ya se mencionó que hay dos tipos de receptores funcionales distintos (ERs), $\alpha$ y $\beta$. Se expresan a nivel del endotelio, plaquetas y en las células del músculo liso. Los ER- $\alpha$ activan directamente la producción del $\mathrm{NO}$ en el endotelio, mediante un efecto no genómico ${ }^{(38)}$. El ER-alfa puede ser considerado como el principal responsable en la producción del NO ${ }^{(39-41)}$.

Los receptores ER-beta ejercen una acción genómica con efecto en general antiaterogénico y antiproliferativo, involucrando también al NO.

\section{Etapas de riesgo vascular en la mujer}

A lo largo de la vida reproductiva de una mujer, se producen cambios relacionados con la edad en el ciclo menstrual, secreción hormonal y fertilidad, que culminan con la menopausia. 
El efecto del ciclo menstrual en la función endotelial hace que esta sea cambiante, y cíclicamente desde la menarquia hasta la menopausia. Aún se desconoce si estos cambios y estímulos conllevan un efecto benéfico. Hay vasodilatación máxima mediada por flujo (dependiente de NO) durante las fases folicular y luteal, correlacionados con niveles altos de estradiol ${ }^{(42)}$.

Durante el climaterio se producen cambios hormonales de gran relevancia y casi abruptos. El descenso de los niveles de estrógenos e inhibinas $\mathrm{A}$ y $\mathrm{B}$ y el aumento de las gonadotropinas FSH y LH son los cambios preponderantes. Cuando se aproxima el último período menstrual, la desaparición de folículos ováricos implica el cese de ovulación con descenso de las inhibinas y del estradiol y aumento de los niveles plasmáticos de FSH. Adicionalmente, hay cambios de los niveles de andrógenos durante la transición menopáusica. Este disbalance entre estrógenos-progestágenos y andrógenos hacen más vulnerable a la mujer para daño vascular y la aparición de la hipertensión arterial (HTA), más de predominio sistólico debido a la rigidez arterial, la cual está mediada por el NO. Este tipo de HTA de predominio sistólico explica el por qué las mujeres hacen más ataque cerebro-vascular (ACV) que los hombres.

La producción y acción de los esteroides sexuales está controlada genéticamente, incluida la enzima aromatasa, que convierte la testosterona en estradiol. Algunos estudios sugieren que la inhibición de dicha enzima tendría un papel importante en la alteración de la presión arterial ${ }^{(43)}$. También hay evidencia de efectos hormonales directos sobre la función endotelial. Por ejemplo, se ha demostrado que el ciclo menstrual modula la función endotelial en mujeres jóvenes, específicamente. Algunos estudios han demostrado que hay un incremento en la vasodilatación dependiente del endotelio cuando la mujer pasa de la etapa folicular a la fase luteal ${ }^{(44)}$, un período en el cual hay una oleada de estrógenos.
Las mujeres sanas tienen mejor función endotelial que los hombres sanos en el mismo grupo etario, lo que se cree es debido a alternancia de ciclos foliculares y luteales a través del $\mathrm{NO}{ }^{(45)}$.

Igualmente, la disfunción endotelial se presenta más precozmente en hombres que en mujeres ${ }^{(46-49)}$. En contraste, al inicio de la menopausia, la mujer tiene una declinación más aguda de la función endotelial, igualando en poco tiempo a la del hombre ${ }^{(50)}$.

En conclusión, desde las etapas embrionarias tempranas de la diferenciación, el genoma inicia y forma una estructura vascular diferente entre hombres y mujeres. Ellas producen más NO mediada por receptores estrogénicos con acciones genómicas y no genómicas y están programadas para cambios cíclicos hormonales que también inciden en la función endotelial.

La mujer tiende a hacer más disfunción microvascular que el hombre, y en la menopausia -por pérdida en la producción del NO- hacen más rigidez arterial, más HTA sistólica (mayor presion de pulso) y, por consiguiente, más accidente cerebrovascular. Los hombres también expresan receptores estrogénicos en su vasculatura y plaquetas, pero con menor densidad.

El conocimiento de estas diferencias estructurales y funcionales de la vasculatura entre sexos, ha replanteado y ha hecho surgir el relativamente nuevo campo de Medicina de Géneros.

Esa visión de bikini de la mujer de cáncer de seno, genitalidad, paridad y fertilidad, está muy lejos de lo que hoy día se ha aprendido y avanzado en el impacto que tienen las diferencias vasculares en la prevalencia, comportamiento diferente, manifestaciones diferentes, respuestas terapéuticas diferentes (las mujeres tienen distribución de grasa diferente, lo cual incide en la farmacodinamia de muchos medicamentos hidrofilicos); hay respuesta diferente al intervencionismo coronario (las mujeres tiene coronarias más delgadas y tortuosas, lo cual repercute negativamen- te en los resultados), y también existe cierta discriminación en el abordaje de los problemas de salud de las mujeres, quienes han estado sub-representadas en todos los estudios de intervención farnacológica, extrapolando datos de hombres en mujeres. Muy seguramente lo anterior explique el por qué no ha habido suficiente impacto en las medidas de prevención y tratamiento sobre mortalidad cerebro-cardio-reno-angiovascular en las mujeres, comparativamente con los hombres ${ }^{(43)}$.

\section{REFERENCIAS BIBLIOGRÁFICAS}

1. Castelli WP. Cardiovascular disease in women. Am J Obstet Gynecol. 1988;158:1553-60, 1566-7.

2. Cooper R, Cutler J, Desvigne-Nickens P, Fortmann SP, Friedman L, Havlik R, Hogelin G, Marler J, Mc Govern P, Morosco G, Mosca L, Pearson T, Stamler J, Stryer D, Thom TI. Trends and disparities in coronary heart disease, stroke, and other cardiovascular diseases in the United States: findings of the national conference on cardiovascular disease prevention. Circulation. 2000;102:3137-47.

3. World health statistics 2006. Geneva: World Health Organization; 2006. Disponible en: www.who.int/ whosis/whostat2006.pdf. Obtenido el 19 dic 2006.

4. Dustan HP. Coronary artery disease in women. Can J Cardiol. 1990;6[Suppl B]:19B-21B.

5. Wenger NK. Coronary heart disease in women: an overview (myths, misperceptions and missed opportunities). En: Wenger NK, Speroff L, Packard $B$, eds. Cardiovascular disease and health in women. Greenwich, CT: LeJacq Communications, 1993:21-9.

6. Federmann DD. The biology of human sex differences. N Engl J Med. 2006;354:1507-14.

7. Moretti M, Phillips M, Abouzeid A, Cataneo R, Greenberg J. Increased breath markers of oxidative stress in normal pregnancy and in preeclampsia. Am J Obstet Gynecol. 2004;190:1184-90.

8. Thompson J, Khalil RA. Gender differences in the regulation of vascular tone.Clin Exp Pharm Physiol. 2003;30:1-15.

9. Taddei $S^{1}$, Virdis A, Ghiadoni L, Mattei P, Sudano I, Bernini G, Pinto S, Salvetti A. Menopause is associated with endothelial dysfunction in women. Hypertension. 1996 Oct;28(4):576-82

10. Reis SE, Holubkov R, Conrad Smith AJ, Kelsey SF, Sharaf BL, Rogers WJ, Reichek N, Rogers WJ, Bairey Merz CN, Sopko G, et al. Coronary microvascular dysfunction is highly prevalent in women with chest pain in the absence of coronary artery disease: results from the NHLBI WISE study. Am Heart J. 2001;141:735-41.

11. Zeiher AM, Drexler H, Saurbier B, Just H. Endothelium-mediated coronary blood flow modulation in humans. Effects of age, atherosclerosis, hypercholesterolemia, and hypertension. J Clin Invest. 1993 Aug;92:652-62.

12. Sadera MA, Celermajer DS. Endothelial function, vascular reactivity and gender differences in the cardiovascular system. Cardiovasc Res. 2002;53:597-604. 
13. McCulloch AI, Randall MD. Sex differences in the relative contributions of nitric oxide and EDHF to agoniststimulated endothelium-dependent relaxations in the rat isolated mesenteric arterial bed. $\mathrm{Br}$ J Pharmacol. 1998;123:1700-6.

14. Ahmed SB, Fisher NDL, Hollenberg NK. Gender and the renal nitric oxide synthase system in healthy humans. Clin J Am Soc Nephrol. 2007;2:926-31.

15. Miller AA, De Silva TM, Jackman KA, Sobey CG. Effect of gender and sex hormones on vascular oxidative stress. Clin Exp Pharmacol Physiol. 2007;34:1037-43.

16. Miller AA, De Silva TM, Jackman KA, Sobey CG. Effect of gender and sex hormones on vascular oxidative stress. Clin Exp Pharmacol Physiol. 2007;34:1037-43.

17. Powers RW, Majors AK, Lykins DL, Sims CJ, Lain KY, Roberts JM. Plasma homocysteine and malondialdehyde are correlated in an age- and genderspecific manner. Metabolism. 2002;51:1433-8.

18. Schulze F, Maas R, Freese R, Schwedhelm E, Silberhorn E, Boger RH. Determination of a reference value for NG, NG-dimethyl-L-arginine in 500 subjects. Eur J Clin Invest. 2005;35:622-6.

19. Polderman KH, Stehouwer CDA, van Kamp GJ, Dekker GA, Verheugt FWA, Gooren LJG. Influence of sex hormones on plasma endothelin levels. Ann Intern Med. 1993;118:429-32.

20. Matoba $T$, Shimokawa $H$, Nakashima M, Hirakawa Y,Mukai Y, Hirano K, Kanaide $\mathrm{H}$, et al. Hydrogen peroxide is an endothelium-derived hyperpolarizing factor in mice. J Clin Invest. 2000;106:1521-30.

21. Pak KJ, Geary GG, Duckles SP, Krause DN. Malefemale differences in the relative contribution of endothelial vasodilators released by rat tail artery. Life Sci. 2002;71:1633-42.

22. White RM, Rivera CO, Davison CA. Nitric oxidedependent and -independent mechanisms account for gender differences in vasodilation to acetylcholine. J Pharmacol Exp Ther. 2000;292:375-80.

23. Miller AA, De Silva TM, Jackman KA, Sobey CG. Effect of gender and sex hormones on vascular oxidative stress. Clin Exp Pharmacol Physiol. 2007 Oct;34(10):1037-43.

24. Geary GG, Krause DN, Duckles SP. Estrogen reduces mouse cerebral artery tone through endothelial NOS- and cyclooxygenase-dependent mechanisms. Am J Physiol Heart Circ Physiol. 2000;279:H511-519.

25. Jun SS, Chen Z, Pace MC, Shaul PW. Estrogen upregulates cyclooxygenase-1 gene expression in ovine fetal pulmonary artery endothelium. J Clin Invest. 1998;102:176-83.

26. Ospina JA, Duckles SP, Krause DN. 17 $\beta$-Estradiol decreases vascular tone in cerebral arteries by shifting COX-dependent vasoconstriction to vasodilation. Am J Physiol Heart Circ Physiol. 2003;285:H241-250.
27. Huang A, Sun D, Wu Z, Yan C, Carroll MA, Jiang $\mathrm{H}$, Falck JR, Kaley G. Estrogen elicits cytochrome P450- mediated flow-induced dilation of arterioles in NO deficiency: role of $\mathrm{Pl} 3 \mathrm{~K}$-akt phosphorylation in genomic regulation. Circ Res. 2004;94:245-52.

28. Woodman OL, Boujaoude M. Chronic treatment of male rats with daidzein and 17 beta-oestradiol induces the contribution of EDHF to endothelium-dependent relaxation. Br J Pharmacol. 2004;141:322-8.

29. Liu MY, Hattori Y, Fukao M, Sato A, Sakuma I, Kanno M. Alterations in EDHF-mediated hyperpolarization and relaxation in mesenteric arteries of female rats in longterm deficiency of oestrogen and during oestrus cycle. Br J Pharmacol. 2001;132:1035-46.

30. Sudhir K, Komesaroff PA. Cardiovascular actions of estrogens in men. J Clin Endocrinol Metab. 1999:84:3411-5.

31. Komesaroff PA, Sudhir SK. Estrogens and human cardiovascular physiology. Reprod Fertil Dev. 2001;13:261-72.

32. Yildiz O, Seyrek M. Vasodilating mechanisms of testosterone. Exp Clin Endocrinol Diabetes. 2007;115:1-6.

33. Yildiz O, Seyrek M, Gul H, Un I, Yildirim V, Ozal E, Uzun M, et al. Testosterone relaxes human internal mammary artery in vitro. J Cardiovasc Pharmacol. 2005;45:580-5.

34. English KM JR, Jones TH, Morice AH, Channer KS. Testosterone acts as a coronary vasodilator by a calcium antagonistic action. J Endocrinol Invest. 2002;25:455-8.

35. Bernini G, Versari D, Moretti A, Virdis A, Ghiadon $\mathrm{L}$, Bardini $\mathrm{M}$, et al. Vascular reactivity in congenital hypogonadal men before and after testosterone replacement therapy. J Clin Endocrinol Metab. 2006 May;91(5):1691-7.

36. Komesaroff PA, Black CVS, Westerman RA. A novel, nongenomic action of estrogen on the cardiovascular system. J Clin Endocrinol Metab. 1998;83:2313-6.

37. New M, Gishel, Timmins BH, Katrina L, Duffy M Stephen J, Tran BH, et al. Long-term estrogen therapy improves vascular function in male to female transsexuals. J Am Coll Cardiol. 1997;29:1437-44.

38. Hodges YK, Tung L, Yan X-D, Graham JD, Horwitz KB, Horwitz LD. Estrogen receptors alpha and beta: prevalence of estrogen receptor beta mRNA in human vascular smooth muscle and transcriptiona effects. Circulation. 2000 Apr 18;101(15):1792-8.

39. Darblade B, Pendaries C, Krust A, Dupont S, Fouque M-J, Rami J, Chambon P, et al. Estradiol alters nitric oxide production in the mouse aorta through the alpha-, but not beta-, estrogen receptor. Circ Res. 2002 Mar 8;90(4):413-9.

40. Muller-Delp JM ${ }^{1}$, Lubahn DB, Nichol KE, Philips BJ, Price EM, Curran EM, Laughlin MH. Regulation of nitric oxide-dependent vasodilation in coronary arteries of estrogen receptor-alpha-deficient mice. Am J Physiol Heart Circ Physiol. 2003;285:H2150-7.
41. Sumi D, Ignarro LJ. Estrogen-related receptor alpha 1 up-regulates endothelial nitric oxide synthase expression. Proc Natl Acad Sci. 2003;100:14451-6.

42. English JL, Jacobs LO, Green G, Andrews TC. Effect of the menstrual cycle on endothelium-dependent vasodilation of the brachial artery in normal young women. Am J Cardiol. 1998;82:256-8.

43. Melgarejo E, Urdinola J. Riesgo cardiovascular de la mujer menopáusica. Ed. Sociedad Colombiana de Cardiologia, FECOLSOG 2011. Ed Panamericana 2012.

44. Miller VM, Mulvagh SL. Sex steroids and endothelial function: translating basic science to clinical practice. Trends Pharmacol Sci. 2007;28:263-70.

45. Hashimoto M, Akishita M, Eto M, Ishikawa M, Kozaki K, Toba K, Sagara Y, Taketani Y, Orimo H, Ouchi $Y$. Modulation of Endothelium-dependent flowmediated dilatation of the brachial artery by sex and menstrual cycle. Circulation. 1995;92:3431-5.

46. Halligan SC, Murtagh B, Lennon RJ, Pumper GM, Mathew V, Higano ST, Lerman A. Effect of Longterm hormone replacement therapy on coronary endothelial function in postmenopausal women. Mayo Clin Proc. 2004;79:1514-20.

47. Sader MA, Celermajer DS. Endothelial function, vascular reactivity and gender differences in the cardiovascular system. Cardiovasc Res. 2002 Feb 15;53(3):597-604.

48. Egashira K, Inou T, Hirooka Y, Kai H, Sugimachi M, Suzuki S, Kuga T, Urabe Y, Takeshita A. Effects of age on endothelium-dependent vasodilation of resistance coronary artery by acetylcholine in humans. Circulation. 1993;88:77-81.

49. Celermajer DS, Sorensen KE, Spiegelhalter DJ, Georgakopoulos D, Robinson J, Deanfield JE. Aging is associated with endothelial dysfunction in healthy men years before the age-related decline in women. J Am Coll Cardiol. 1994 Aug;24(2):471-6.

50. Deanfield J, Donald A, Ferri C, Giannattasio C, Halcox J, Halligan S, Lerman A, Mancia G, Oliver $J J$, Pessina AC, et al. Endothelial function and dysfunction. Part I: Methodological issues for assessment in the different vascular beds: A statement by the Working Group on Endothelin and Endothelial Factors of the European Society of Hypertension. J Hypertens. 2005;23:7-17.

Conflictos de interés:

El autor declara no haber conflictos de interés.

Correspondencia:

Correo electrónico: enrique.melgarejo@gmail.com 